\title{
Surface chemistry in photodissociation regions (Corrigendum)
}

\author{
G. B. Esplugues ${ }^{1}$, S. Cazaux ${ }^{1}$, R. Meijerink ${ }^{2}$, M. Spaans ${ }^{1}$, and P. Caselli ${ }^{3}$ \\ 1 Kapteyn Astronomical Institute, University of Groningen, PO Box 800, NL 9700 AV Groningen, The Netherlands \\ e-mail: esplugues@astro.rug.nl \\ 2 Leiden Observatory, Leiden University, PO Box 9513, NL 2300 RA Leiden, The Netherlands \\ 3 Max Planck Institute for Extraterrestrial Physics, Giessenbachstrasse 1, 85748 Garching, Germany
}

A\&A 591, A52 (2016), DOI: 10.1051/0004-6361/201528001

Key words. astrochemistry - ISM: abundances - photon-dominated region (PDR) - errata, addenda

\section{Introduction}

In Table A.5 (page 17) of our original publication (Esplugues et al. 2016), the rate coefficient considered for the $\mathrm{CO}$ ice photodesorption was $2.2 \times 10^{-15} \mathrm{~s}^{-1}$, however we should have considered a coefficient of $3.67 \times 10^{-10} \mathrm{~s}^{-1}$ according to recent results (Fayolle et al. 2011; Muñoz-Caro et al. 2016). We also update here the values for the solid species $\mathrm{H}_{2} \mathrm{O}$ and $\mathrm{H}_{2} \mathrm{CO}$ considering a coefficient of $3.67 \times 10^{-11} \mathrm{~s}^{-1}$ for both of them (see Table 1) instead of $2.16 \times 10^{-11} \mathrm{~s}^{-1}$. In particular, the photoprocess reaction rate, $R_{\text {photo }}\left(\mathrm{cm}^{-3} \mathrm{~s}^{-1}\right)$, is calculated for these cases as

$R_{\text {photo }}=n_{i} f_{\text {ss }} k_{\text {photo }}$,

where $n_{i}$ is the number density of the photodissociated species, $f_{\mathrm{ss}}$ is the self-shielding factor, and $k_{\text {photo }}\left(\mathrm{s}^{-1}\right)$ is the photoprocess rate coefficient as follows:

$$
\begin{aligned}
k_{\text {photo }} & =\frac{\chi F_{\text {Draine }}}{4 n_{\text {surf }} N_{\text {lay }}} Y_{i} \\
& \simeq 2.16 \times 10^{-8} \chi Y_{i}=3.67 \times 10^{-8} G_{0} Y_{i}=\alpha_{i} G_{0},
\end{aligned}
$$

following Chaparro-Molano \& Kamp (2012). In expression (2), $\chi$ is the UV field strength (Draine 1978) ${ }^{1}$, and the photon flux produced by this field per unit area is $F_{\text {Draine }}=$ $1.921 \times 10^{8} \mathrm{~cm}^{-2} \mathrm{~s}^{-1}$ (Woitke et al. 2009). Furthermore, $n_{\text {surf }}=$ $1.11 \times 10^{15} \mathrm{~cm}^{-2}$ is the surface density of available absorption sites per unit grain area assuming $3 \AA$ separation between sites, $N_{\text {lay }}=2$ is the assumed number of ice layers that photons can penetrate for photodesorption (Andersson et al. 2006; Arasa et al. 2010; Muñoz-Caro et al. 2016), and $Y_{i}$ is the photodesorption yield per photon (see Table 2).

These corrections lead to variations in some of the results included in the original publication. The variations are mainly produced at visual extinctions $A_{\mathrm{V}} \gtrsim 4$ mag. In particular, significant differences are found for Model $1\left(n_{\mathrm{H}}=10^{4} \mathrm{~cm}^{-3}\right.$, $\left.G_{0}=10^{4}\right)$, while results for Model $2\left(n_{\mathrm{H}}=10^{6} \mathrm{~cm}^{-3}, G_{0}=10^{4}\right)$ and Model $3\left(n_{\mathrm{H}}=10^{6} \mathrm{~cm}^{-3}, G_{0}=10^{2}\right)$ are barely affected. We

\footnotetext{
1 Draine field $(\chi) \simeq 1.7 \times$ Habing field $\left(\mathrm{G}_{0}\right)$.
}

Table 1. Photoreactions on dust grains.

\begin{tabular}{ll}
\hline \hline Reactions $^{a}$ & $\alpha_{i}\left(\mathrm{~s}^{-1}\right)$ \\
\hline $\mathrm{J}(\mathrm{CO})+$ Photon $\rightarrow \mathrm{CO}$ & $3.67 \times 10^{-10}$ \\
$\mathrm{~J}\left(\mathrm{H}_{2} \mathrm{CO}\right)+$ Photon $\rightarrow \mathrm{H}_{2} \mathrm{CO}$ & $3.67 \times 10^{-11}$ \\
$\mathrm{~J}\left(\mathrm{H}_{2} \mathrm{O}\right)+$ Photon $\rightarrow \mathrm{H}_{2} \mathrm{O}$ & $3.67 \times 10^{-11}$ \\
\hline
\end{tabular}

Notes. ${ }^{(a)}$ The expression $\mathrm{J}(i)$ means ice of the species $i$.

Table 2. Photoreaction yields.

\begin{tabular}{lll}
\hline \hline Species & Yield & Reference \\
\hline $\mathrm{CO}$ & $1 \times 10^{-2}$ & Fayolle et al. (2011) and references therein \\
$\mathrm{H}_{2} \mathrm{CO}$ & $1 \times 10^{-3}$ & Guzmán et al. (2013) \\
$\mathrm{H}_{2} \mathrm{O}^{a}$ & $f(x, T) \times 10^{-3}$ & Öberg et al. (2009b) \\
\hline
\end{tabular}

Notes. ${ }^{(a)}$ Here, $f(x, T)=(1.3+0.032 \times T)\left(1-\mathrm{e}^{-x / l(T)}\right)$. At low dust temperatures $(<15 \mathrm{~K})$, the photodesorption yield is $\sim 1 \times 10^{-3}$ for ice thickness between 1 and 10 monolayers.

show the new figures for those cases affected by the new rate coefficients below. We also compare these new figures with those from the original paper. In spite of these variations, all the conclusions obtained in Esplugues et al. (2016) remain the same.

\section{Cooling}

The corrected version of the paper, Fig. 1 (left), shows that cooling by $\mathrm{CO}$ becomes dominant at $3<A_{\mathrm{V}} \lesssim 5$ mag. In the original paper (right), the cooling is dominated by $\mathrm{CO}$ and [OI] $63 \mu \mathrm{m}$ at $3<A_{\mathrm{V}} \lesssim 4$ mag and by gas-grain collisions at $4<A_{\mathrm{V}} \lesssim 5$ mag.

\section{Chemical structure}

\subsection{Gas-phase species}

The corrected version of the paper, Fig. 2 (top), shows high CO gas-phase abundances in comparison with those obtained in the 

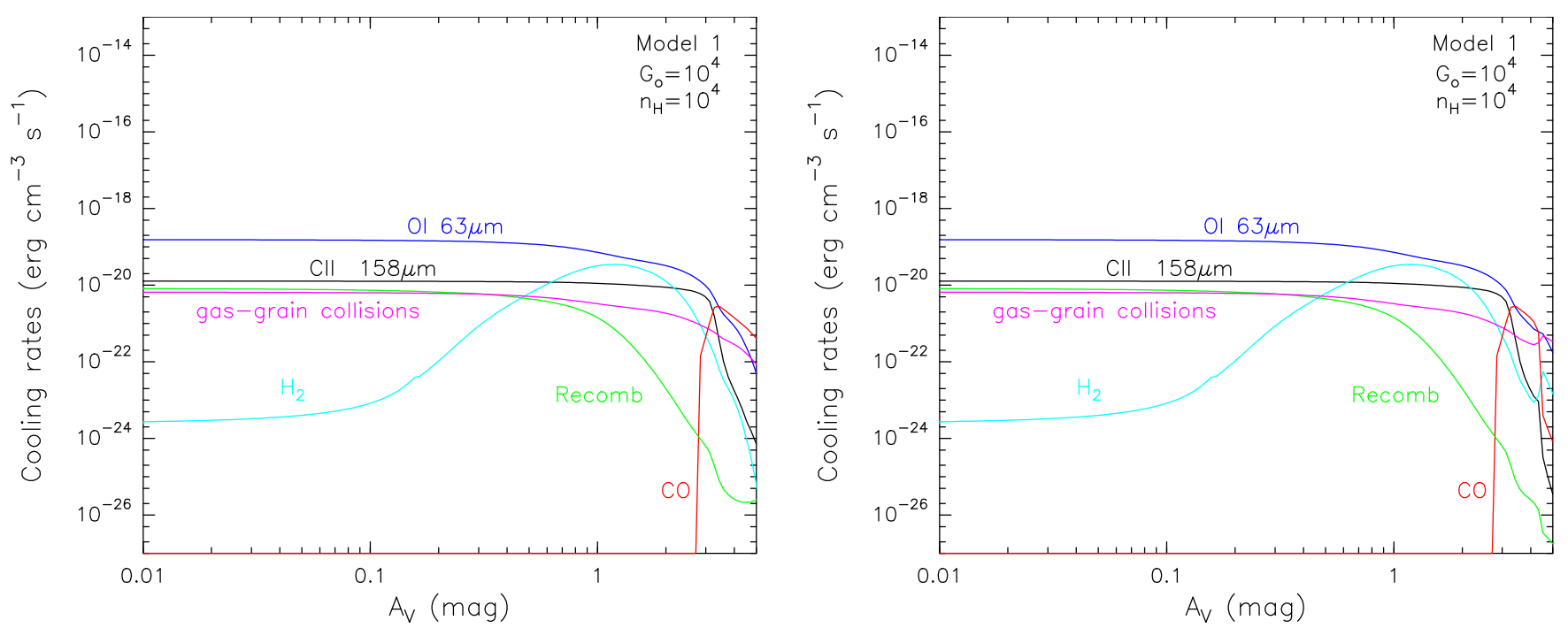

Fig. 1. Cooling processes for Model 1 as calculated in the original paper (right) and after the rate coefficient correction (left).
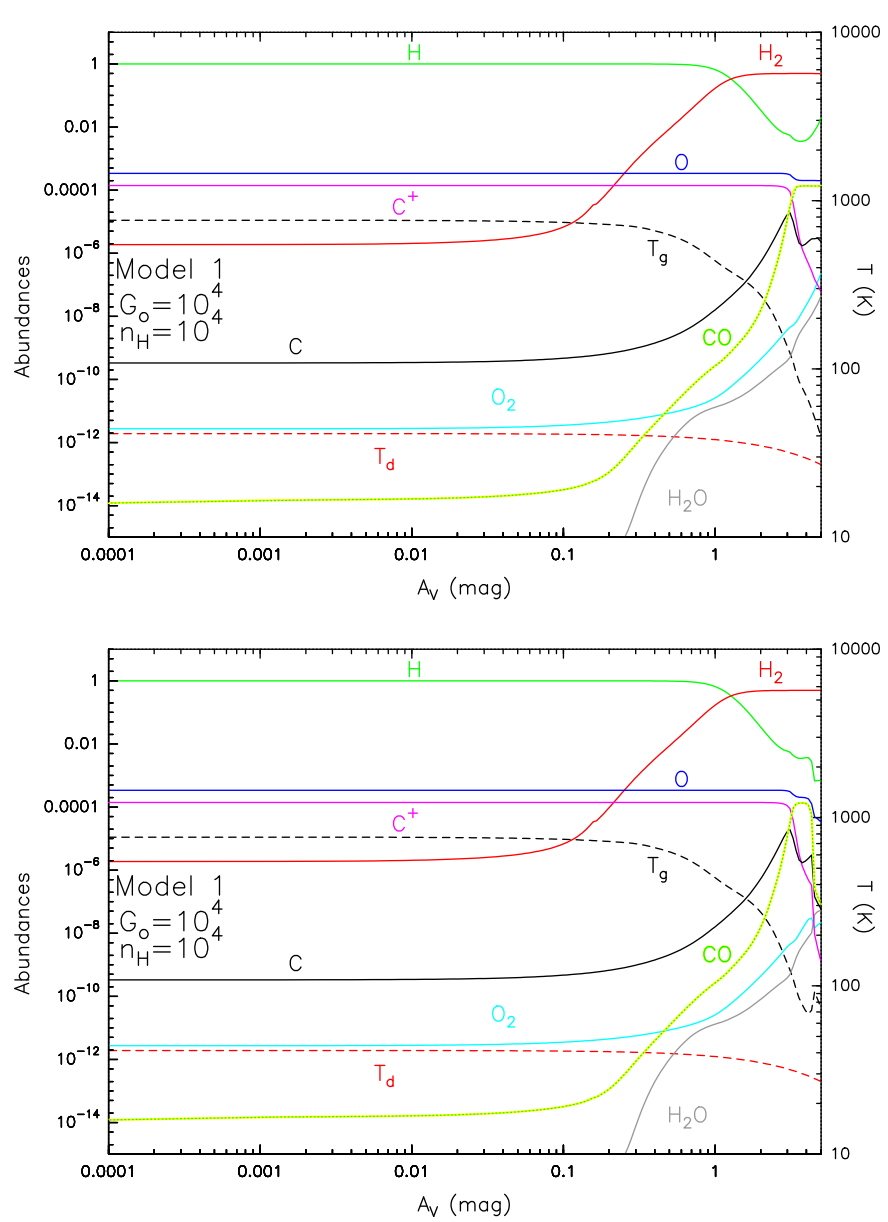

Fig. 2. Gas-phase fractional abundances, $n(\mathrm{x}) / n_{\mathrm{H}}$, for Model 1 as calculated in the original paper (bottom) and after the rate coefficient correction (top).

original paper (bottom) at $4 \lesssim A_{\mathrm{V}} \lesssim 5$ mag. For this visual extinction range, the gas temperature is also lower in the corrected version due to the higher $\mathrm{CO}$ contribution to the cooling of the region. The abundances of the other species showed in Fig. 2 present insignificant differences (less than one order of magnitude) between both plots.

\subsection{Dust-phase species}

Figure 3 (left) shows that, taking the updated rate coefficients into account, one full monolayer of $\mathrm{CO}_{2}$ and $\mathrm{H}_{2} \mathrm{O}$ ice at $A_{\mathrm{V}} \lesssim$ 5 mag does not form for Model 1, unlike the original (right) paper (see also Fig. 4 with the exact number of monolayers formed in each case). We also find a significant difference in the abundances of solid $\mathrm{H}_{2} \mathrm{O}_{2}$. In particular, with the new photodesorption rate coefficients (left), the $\mathrm{H}_{2} \mathrm{O}_{2}$ abundances remain high at $4<A_{\mathrm{V}} \lesssim 5 \mathrm{mag}$ in comparison with the original publication (right).

\subsection{Ice species formation rates}

For chemical reactions forming water ice (Fig. 5), the main difference between the original paper (right) and the corrected version (left) is found in the rates of the reaction between solid $\mathrm{H}$ and solid $\mathrm{H}_{2} \mathrm{O}_{2}$ for Model 1 at large $A_{\mathrm{V}}$. In spite of this difference, however, we still obtain that the main chemical reaction forming water ice at $A_{\mathrm{V}}>4$ mag for Model 1 is the reaction between solid $\mathrm{H}$ and solid $\mathrm{OH}$.

Figure 6 shows the rates of the reactions forming $\mathrm{CO}_{2}$ ice in the original paper (right) and in the corrected version (left). The main differences between both plots are found once the maximum number of $\mathrm{CO}_{2}$ monolayers is reached (at $\sim 3.5 \mathrm{mag}$ and $\sim 1.5$ mag for Models 2 and 3, respectively). For these cases, the $\mathrm{CO}_{2}$ ice formation is dominated by the reaction of solid $\mathrm{CO}$ with solid $\mathrm{O}$ and solid $\mathrm{OH}$. For Model 1, in the corrected version, we obtain that $\mathrm{CO}_{2}$ is mainly formed only through the reaction between solid $\mathrm{CO}$ and solid $\mathrm{O}$ at $A_{\mathrm{V}} \lesssim 5 \mathrm{mag}$. In the original version of the paper, however, we obtain that $\mathrm{CO}_{2}$ ice is formed through solid $\mathrm{OH}$ and solid $\mathrm{CO}$ at $4.5 \lessgtr A_{\mathrm{V}} \lesssim 5 \mathrm{mag}$.

\section{Desorption probabilities}

Figure 7 shows abundances for several gas-phase species considering two distinct desorption probabilities $\left(\delta_{\text {ice }}\right)$. The main 
G. B. Esplugues et al.: Surface chemistry in photodissociation regions
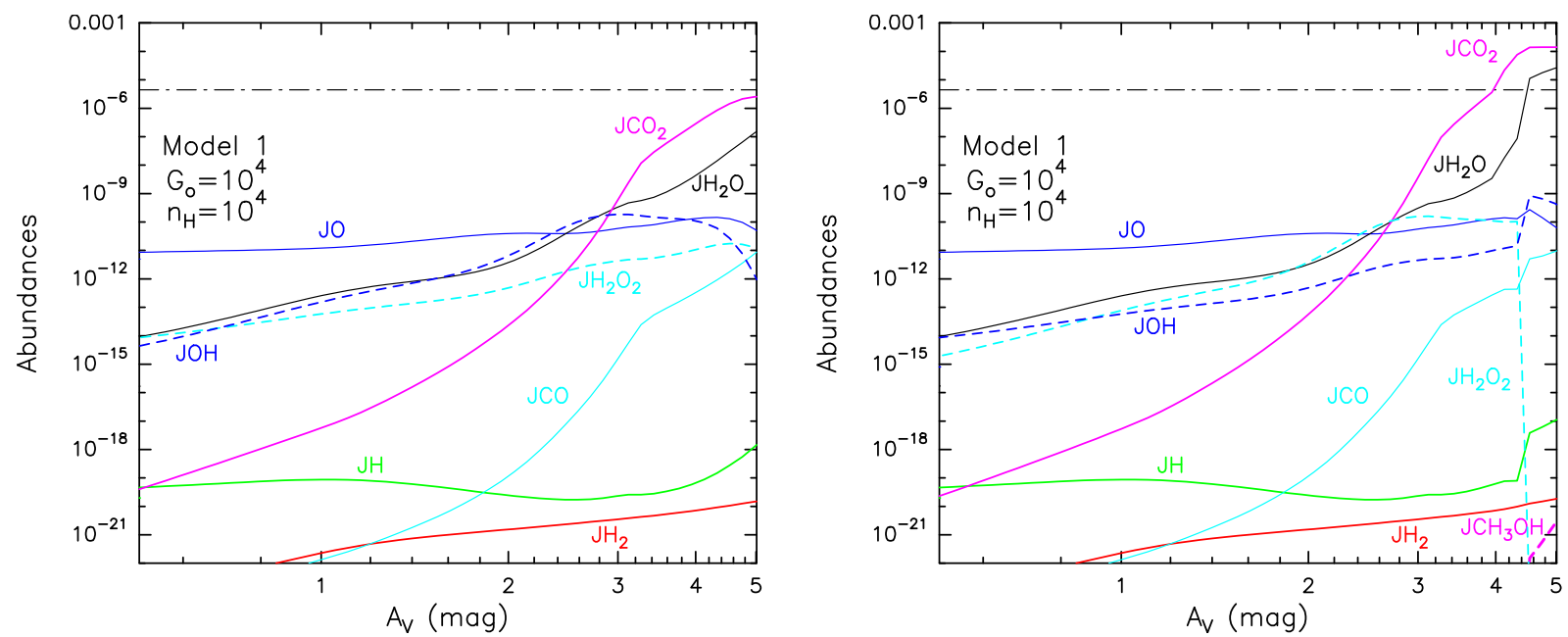

Fig. 3. Dust-phase fractional abundances, $n(\mathrm{x}) / n_{\mathrm{H}}$, for Model 1 as calculated in the original paper (right) and after the rate coefficient correction $(l e f t)$. The dash-dotted black line represents the number of possible attachable sites on grain surfaces per $\mathrm{cm}^{3}$. JX means solid X.
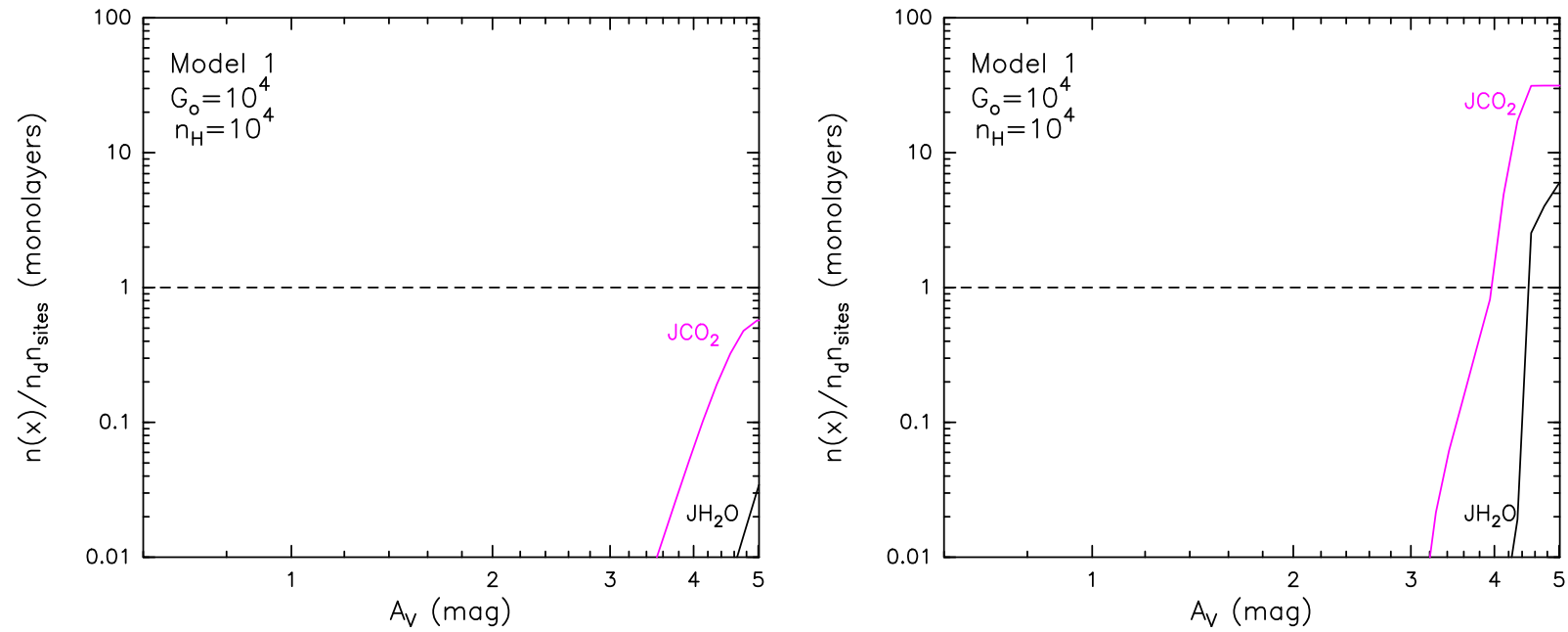

Fig. 4. Growth of ice layers on grains surfaces for $\mathrm{H}_{2} \mathrm{O}$ and $\mathrm{CO}_{2}$ in the original paper (right) and in the corrected version (left). JX means solid X.
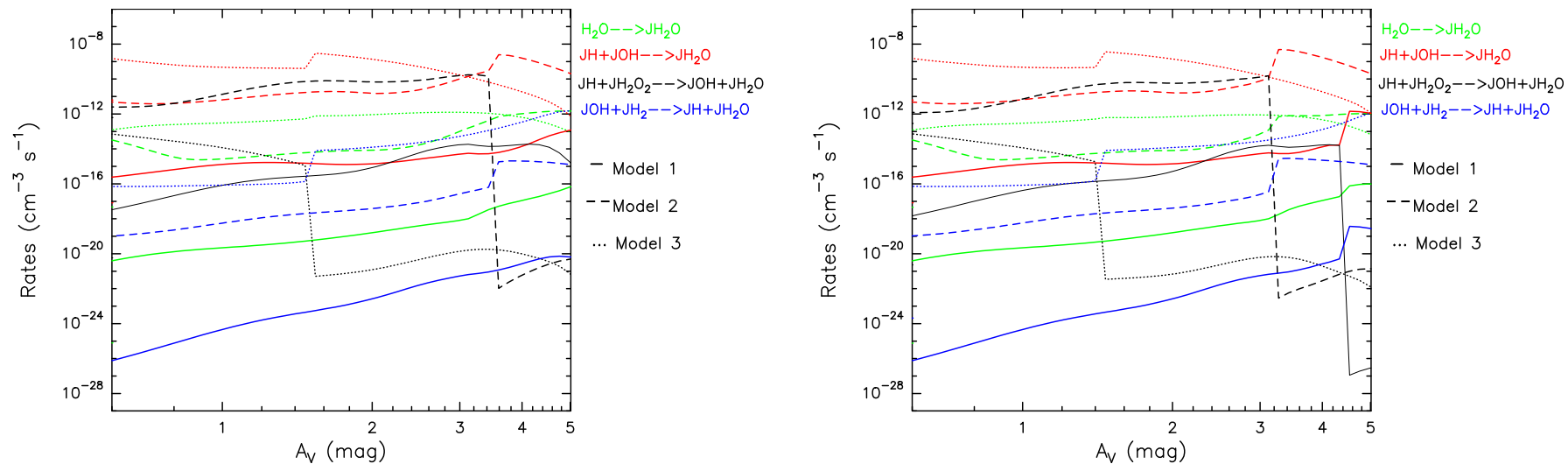

Fig. 5. Rates for surface reactions forming $\mathrm{H}_{2} \mathrm{O}$ ice in the original paper (right) and in the corrected version (left). JX means solid X.

differences between both plots are found for $\mathrm{CO}$ at large visual extinctions $\left(A_{\mathrm{V}}>4 \mathrm{mag}\right)$. In particular, while the $\mathrm{CO}$ abundances drop at $A_{\mathrm{V}} \sim 4$ mag in the original paper (right), this drop occurs deeper in the cloud $\left(A_{\mathrm{V}} \sim 6 \mathrm{mag}\right)$ in the corrected version (left). It leads to a difference of $\sim 3$ orders of magnitude in the $\mathrm{CO}$ abundances between both $\delta_{\text {ice }}$ in the corrected version of the paper. For $\mathrm{H}_{2} \mathrm{CO}$ and $\mathrm{CH}_{3} \mathrm{OH}$, we still obtain differences of up to two and three orders of magnitude, respectively, between both $\delta_{\text {ice }}$ in the corrected paper as in the original publication. 

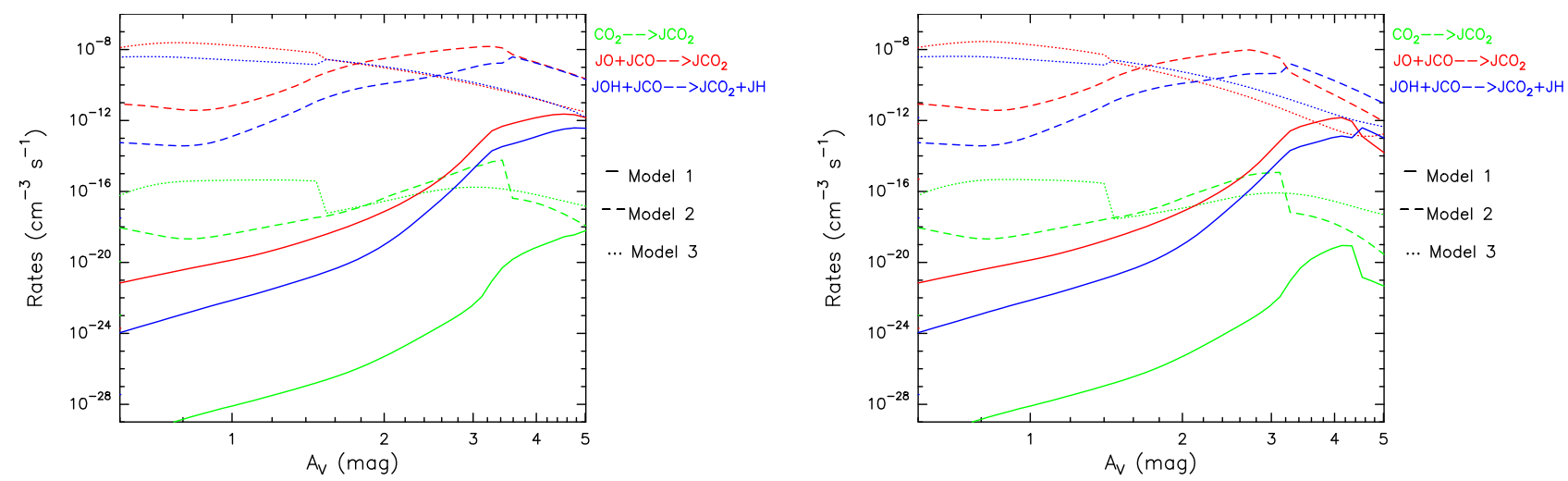

Fig. 6. Rates for surface reactions forming $\mathrm{CO}_{2}$ ice in the original paper (right) and in the corrected version (left). JX means solid X.
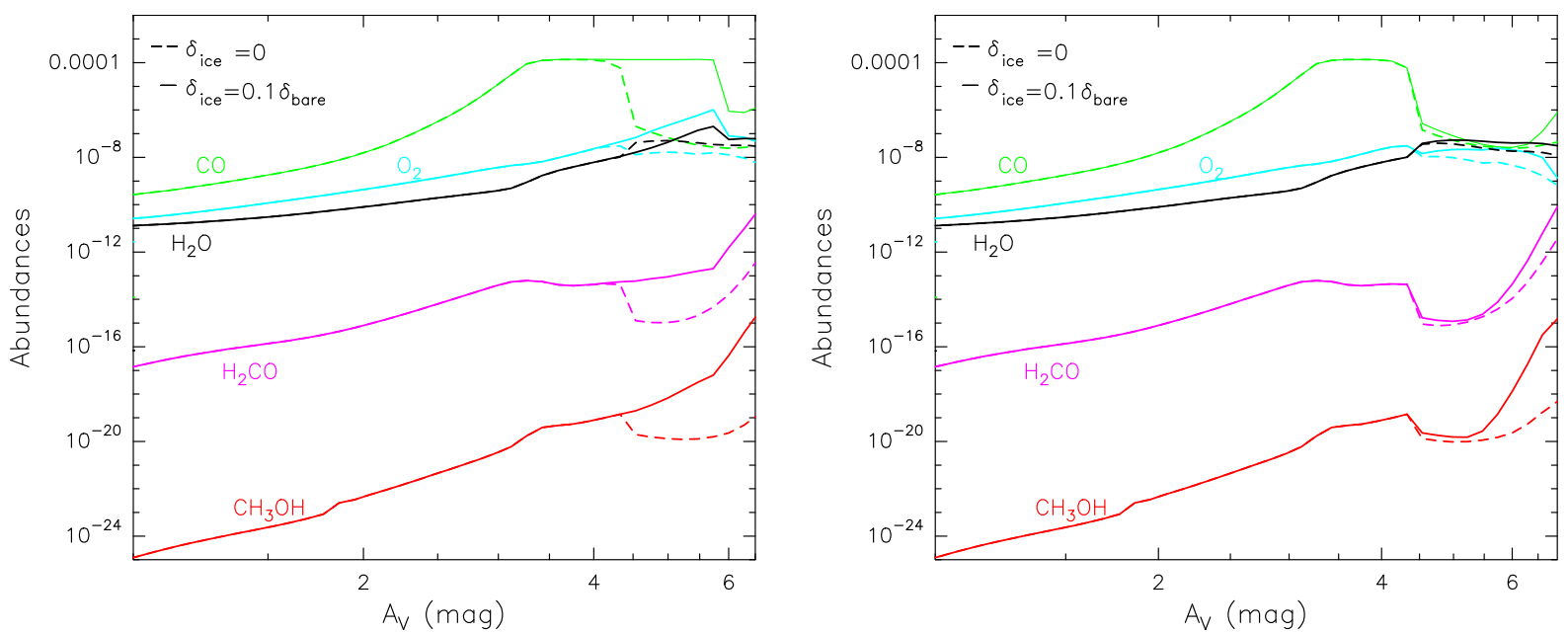

Fig. 7. Gas-phase fractional abundances, $n(\mathrm{x}) / n_{\mathrm{H}}$, for Model $1\left(G_{0}=10^{4}\right.$ and $\left.n_{\mathrm{H}}=10^{4}\right)$ considering different desorption probabilities $\left(\delta_{\text {ice }}\right)$. Original paper (right) and corrected version (left).
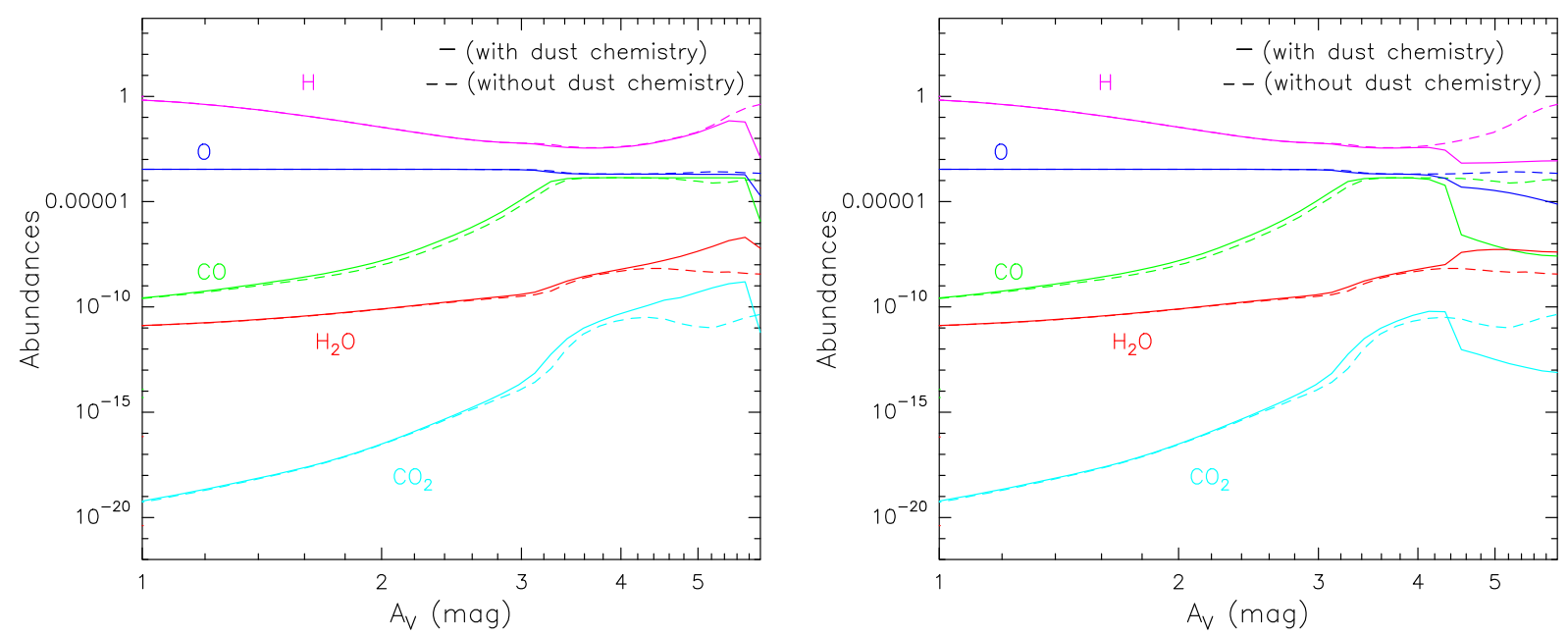

Fig. 8. Gas-phase fractional abundances, $n(\mathrm{x}) / n_{\mathrm{H}}$, for $\mathrm{H}, \mathrm{O}, \mathrm{CO}, \mathrm{H}_{2} \mathrm{O}$, and $\mathrm{CO}_{2}$ from Model $1\left(G_{0}=10^{4}\right.$ and $\left.n_{\mathrm{H}}=10^{4}\right)$ with and without considering dust chemistry. Original paper (right) and corrected version (left).

\section{Effect of dust in the chemical composition of PDRs}

Figures 8 and 9 show a comparison of gas-phase abundances for several species with and without dust chemistry between the original (right) and the corrected paper (left). The main differences are found for $\mathrm{CO}, \mathrm{CO}_{2}, \mathrm{HCO}^{+}$, and $\mathrm{CH}_{3} \mathrm{OH}$. In the corrected version of the paper, the abundances of these species are higher with dust chemistry than without dust chemistry at $4<A_{\mathrm{V}}<6$ mag, unlike the original paper. 
G. B. Esplugues et al.: Surface chemistry in photodissociation regions
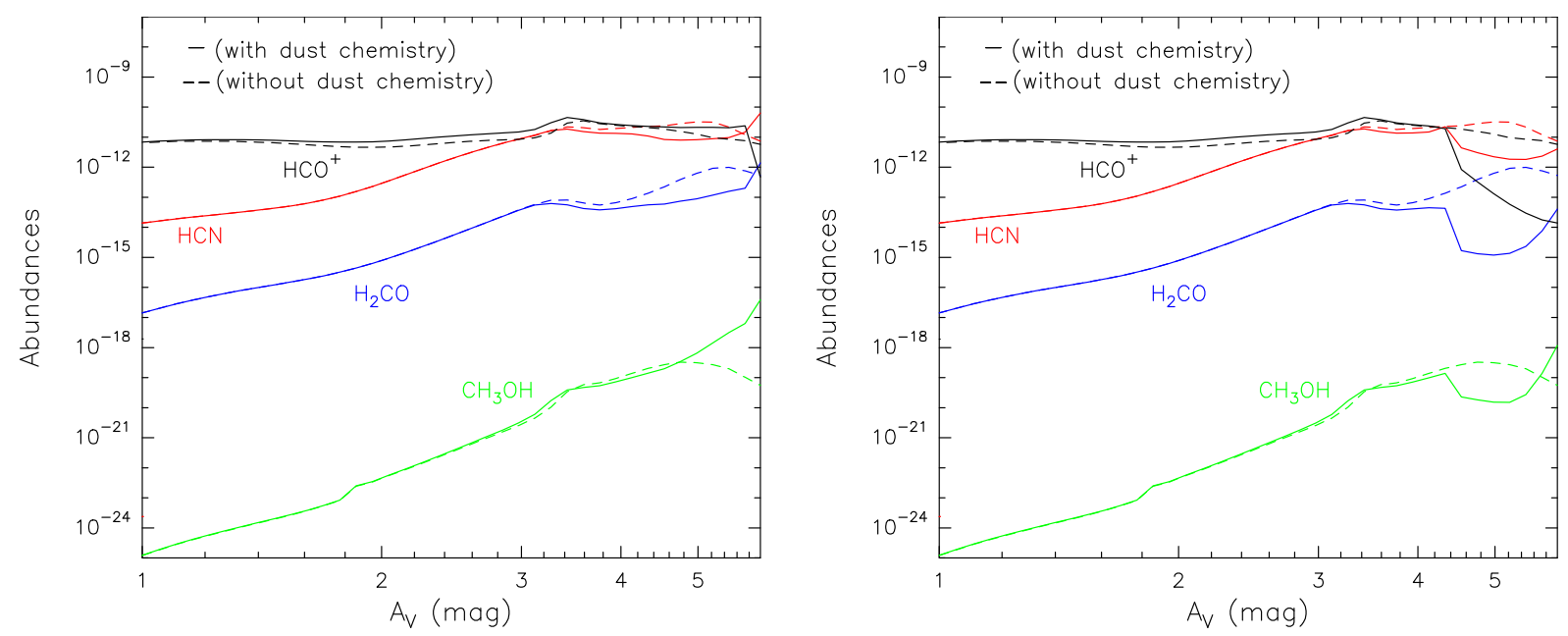

Fig. 9. Gas-phase fractional abundances, $n(\mathrm{x}) / n_{\mathrm{H}}$, for $\mathrm{HCO}^{+}, \mathrm{HCN}, \mathrm{H}_{2} \mathrm{CO}$, and $\mathrm{CH}_{3} \mathrm{OH}$ from Model $1\left(G_{0}=10^{4}\right.$ and $\left.n_{\mathrm{H}}=10^{4}\right)$ with and without considering dust chemistry. Original paper (right) and corrected version (left).
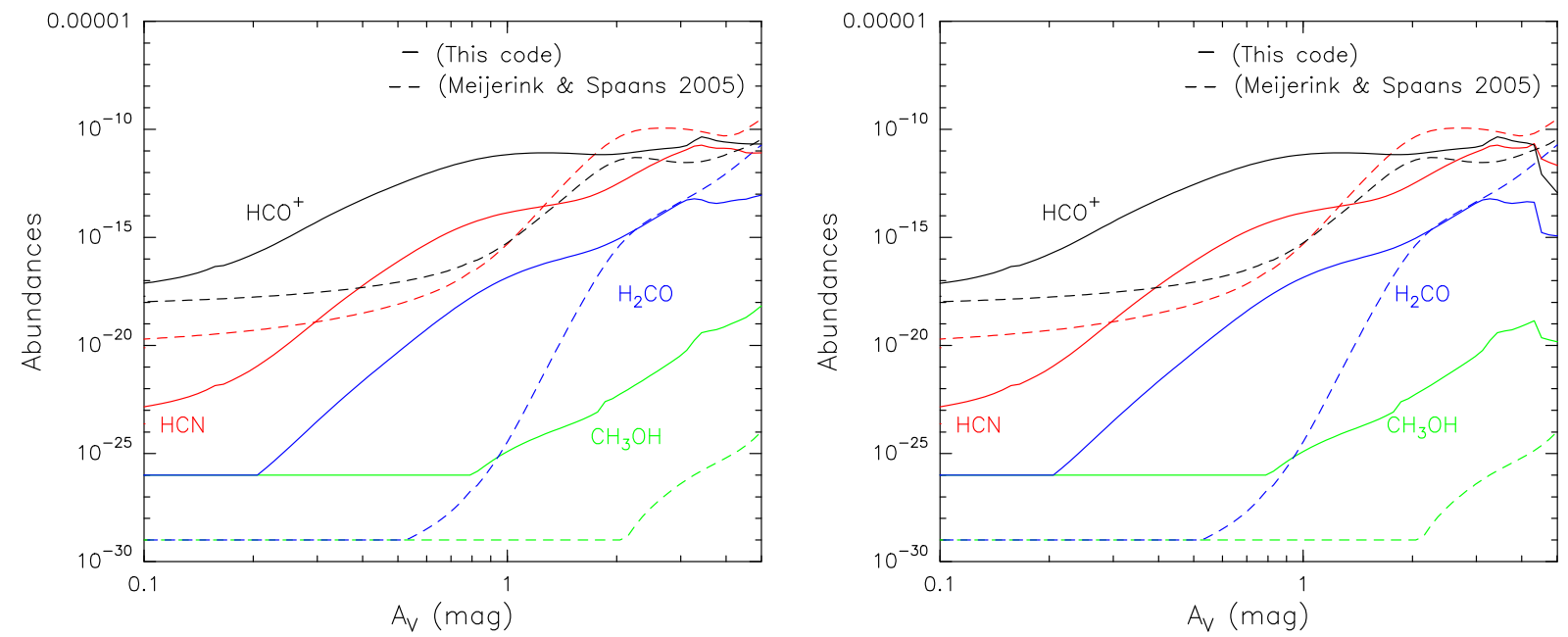

Fig. 10. Gas-phase fractional abundances, $n(\mathrm{x}) / n_{\mathrm{H}}$, for $\mathrm{HCO}^{+}, \mathrm{HCN}, \mathrm{H}_{2} \mathrm{CO}$, and $\mathrm{CH}_{3} \mathrm{OH}$ from Model $1\left(G_{0}=10^{4}\right.$ and $\left.n_{\mathrm{H}}=10^{4}\right)$ obtained with this PDR code and the version from Meijerink \& Spaans (2005). Original paper (right) and corrected version (left).
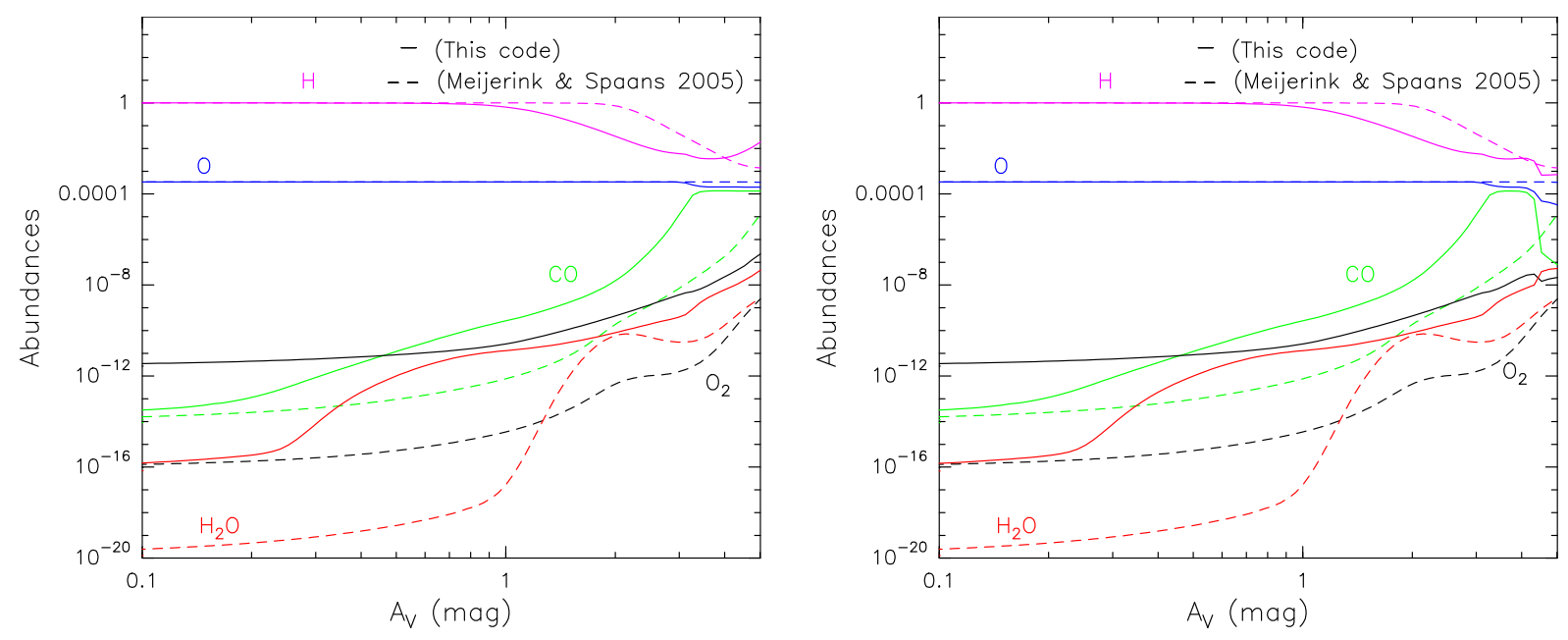

Fig. 11. Gas-phase fractional abundances, $n(\mathrm{x}) / n_{\mathrm{H}}$, for $\mathrm{H}, \mathrm{O}, \mathrm{CO}, \mathrm{H}_{2} \mathrm{O}$, and $\mathrm{O}_{2}$ from Model $1\left(G_{0}=10^{4}\right.$ and $\left.n_{\mathrm{H}}=10^{4}\right)$ obtained with this PDR code and the version from Meijerink \& Spaans (2005). Original paper (right) and corrected version (left). 


\section{Comparison with the original Meijerink PDR code}

Figures 10 and 11 show gas-phase abundances for several species obtained with this PDR code and with the version from Meijerink \& Spaans (2005) for the original paper (right) and the corrected paper (left). In the corrected version, the abundances of $\mathrm{HCO}^{+}, \mathrm{H}_{2} \mathrm{CO}$, and $\mathrm{CH}_{3} \mathrm{OH}$ (Fig. 10) are up to two orders of magnitude larger than in the original publication at $4 \lesssim A_{\mathrm{V}} \lesssim 5$ mag. In Fig. 11, we find the main difference in the $\mathrm{CO}$ abundances between the original paper (right) and the corrected version (left) at $4 \lesssim A_{\mathrm{V}} \lesssim 5$ mag. Other species shown in Fig. 11 present differences that are lower than one order of magnitude at $4 \lesssim A_{\mathrm{V}} \lesssim 5 \mathrm{mag}$. For lower visual extinctions, all the abundances remain unchanged.
Acknowledgements. The authors thank Evelyne Roueff for pointing out the error of the rate coefficient for the $\mathrm{CO}$ photodesorption in our original publication.

\section{References}

Andersson, S., Al-Halabi, A., Kroes, G. J., \& van Dishoeck, E. F. 2006, J. Chem. Phys., 124, 064715

Arasa, C., Andersson, S., Cuppen, H. M., et al. 2010, J. Chem. Phys., 132, 184510

Chaparro-Molano, G., \& Kamp, I. 2012, A\&A, 537, A138

Draine, B. T. 1978, ApJS, 36, 595

Esplugues, G. B., Cazaux, S., Meijerink, R., et al. 2016, A\&A, 591, A52

Fayolle, E. C., Bertin, M., Romanzin, C., et al. 2011, ApJ, 739, L36

Guzmán, V., Goicoechea, J. R., Pety, J., et al. 2013, A\&A, 560, A73

Meijerink, R., \& Spaans, M. 2005, A\&A, 436, 397

Muñoz-Caro, G. M., Chen, Y. J., Aparicio, S., et al. 2016, A\&A, 589, A19

Öberg, K., Linnartz, H., Visser, R., \& van Dishoeck, E. F. 2009b, ApJ, 693, 1209

Woitke, P., Kamp, I., \& Thi W. F. 2009, A\&A, 501, 383 